\title{
Primary palliative care. Caring for patients with life-limiting illness in the community
}

Sylvia McCarthy

McCarthy S. Primary palliative care. Caring for patients with life-limiting illness in the community. Malays Fam Physician. 2021;16(3);2-5.

https://doi.org/10.51866/cm0002

\section{Keywords:}

Palliative care, community, primary care

\section{Authors:}

\section{Sylvia McCarthy}

Medical Director

Hospis Malaysia

2, Jalan 4/96, Off Jalan Sekuci

Jn Cheras, Taman Sri Bahtera

Cheras, Kuala Lumpur, Malaysia

\section{Abstract}

The 9th October 2021, was World Palliative Care Day. This year's theme for world palliative care is "Leave No One Behind - Equity in Access to Palliative Care".

Evidence for the outcomes of early palliative care is growing. In 2014, the World Health Assembly passed a resolution that was co-sponsored by Malaysia. The resolution called for countries to improve access to palliative care as a core component of health systems, with an emphasis on primary health care and community/home-based care. ${ }^{1}$

One study conducted in Malaysia in 2019 estimated that by 2030, with the increase in noncommunicable diseases, 246000 patients would require palliative care. For Malaysia to achieve equity in access to palliative care, care for these patients must be integrated into primary care. This article discusses some of the tools available for early identification of patients assessment and management of patients with palliative care needs.

Early palliative care can reduce emergency admissions and support patients to die in their preferred place. $^{2,3}$ Increasing evidence supports the need for early palliative care for patients with organ failure, frailty, and neurodegenerative diseases. ${ }^{4}$

For patients with organ failure, prognostication is inaccurate; with any exacerbation, the outcome may be either sudden death or recovery but with less function than before. The palliative care Bowtie Model (Figure 1) has been proposed to describe the integration of palliative care and disease management across the illness trajectory. 5 This integrated model allows patients and their doctors to plan for their deterioration and end of life while maintaining hope for improvement and good quality of life.

Well-being trajectories show the changes in four dimensions - physical, psychological, social and spiritual - over the course of a life-limiting illness and help health care professionals establish where and when patients need more support (Figure 2). ${ }^{6}$

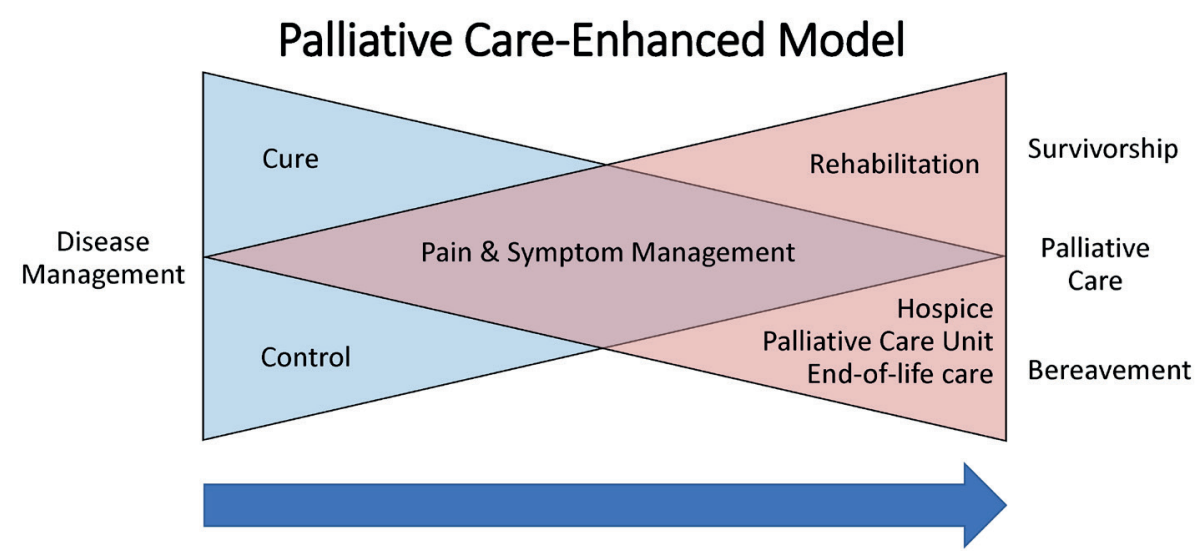

Figure 1. The palliative care Bowtie Model. 


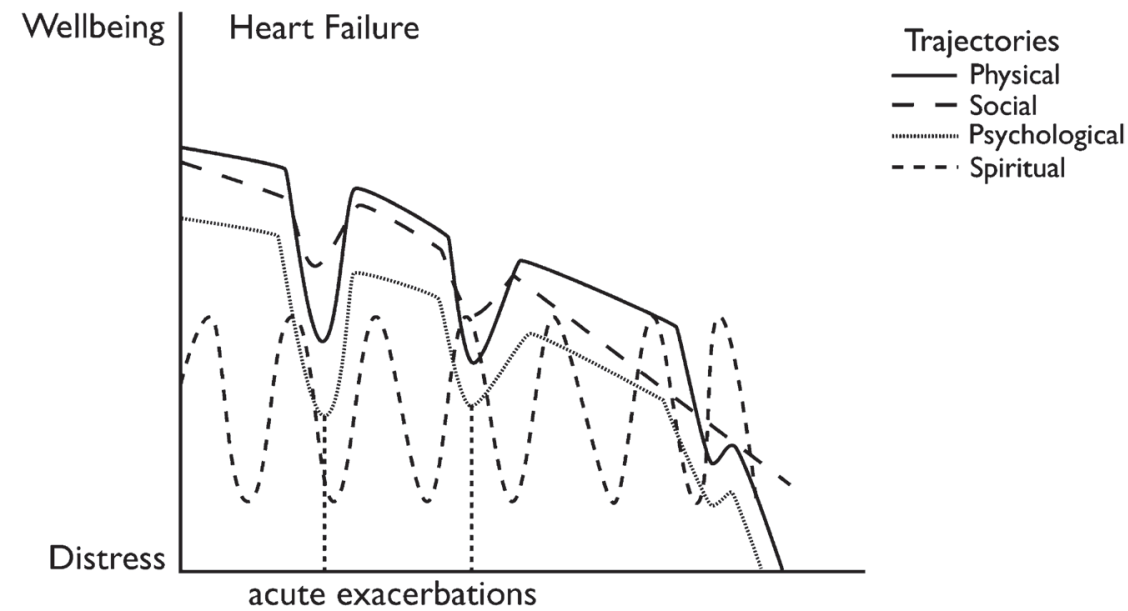

Figure 2. Four dimensions of wellbeing trajectories.

Community palliative care often refers to the care provided by community palliative care teams who provide specialised services for patients at home. However, these services cannot meet the need for early palliative care. To address this gap, a new definition of Primary palliative care has been proposed ${ }^{7}$; see box 1 .

Box 1. Definition of primary palliative care.

Primary palliative care is palliative care practised by primary health care workers, who are the principal providers of integrated health care for people in local communities throughout their life. It includes early identification and triggering of palliative care as part of integrated and holistic chronic disease management, collaborating with specialist palliative care services where they exist, and strengthening underlying professional capabilities in primary care.

This definition recognises that primary care physicians are ideally placed to identify patients living with serious illness who may be suffering from the impact of illness and for whom focusing on quality of life outcomes are now more important than disease outcomes.

Several tools have been developed to help primary physicians identify patients with palliative care needs. One of these is the Supportive and Palliative Care Indicator Tool ${ }^{8}$ or SPICT, which has been developed for use in primary care settings to help primary care physicians identify patients under their care who may have palliative care needs. Rather than identifying specific needs, the SPICT identifies those patients who would benefit from further assessment and care planning. The areas where SPICT can help are outlined in box (2) below.

\section{Box 2. Benefits of Using SPICT.}

- Offers people opportunities to talk about their health declining and 'what matters' to them, including things they hope to do, would like to happen, or wish to avoid.

- Gives priority to maintaining a person's quality of life, and involving the people who are close to them.

- Ensures that any available disease-modifying treatments of benefit are combined with good symptom control and other aspects of palliative care.

- Reduces the risk of complex treatment and care decisions in a 'crisis' by making emergency care plans for acute illness or complications (including for family caregiver changes).

- Encourages people to appoint a proxy decision-maker (Power of Attorney) and/or make 'advance decisions' about any treatments they do not want in the future.

- Improves communication, continuity of care, current and future care planning,; and multidisciplinary teamwork.

- Includes family members and other carers so they receive the help and support they need. 
Assessment of palliative care needs requires a patient-centred, holistic approach to care. These are core features of family medicine.

What are "palliative care needs"? There is no unified definition, and the needs will be different for each patient. Palliative care needs can be considered in four quadrants: physical, psychological, social and spiritual. For individual patients, these areas cannot be addressed separately; each dimension impacts the others, and holistic care depends on understanding the interplay between them. However, the four quadrants help offer structure to an assessment of palliative care needs. Various tools are available to support palliative assessment. In the physical quadrant, patient-reported outcome measures such as the IPOS $^{9}$ and ESAS $^{10}$ aid with identifying symptoms and symptom severity. These tools are simple to use and include questions about mood and well-being.

The distress thermometer, which was developed in cancer care, is a useful screening tool for distress and identifying the factors causing the distress. ${ }^{11}$

Box 3. Definition of spirituality in palliative care.
Many health care professionals are concerned about addressing issues of spirituality because of religious sensitivities. However spirituality is much broader than this, it encompasses how patients make meaning of their lives, their values, human connections and purpose. A definition of spirituality in palliative care was proposed in 2009. ${ }^{12}$ See box 3 .

How do we support patients emotionally and spiritually? Harvey Chochinov provides an excellent guide in his description of $\mathrm{ABCD}$ (Attitude, Behaviour, Compassion, Dialogue) of Dignity Conserving Care. ${ }^{13}$ These simple steps can ensure that patients are treated with dignity, which is core to supporting their needs. The Patient Dignity Question can help us elicit personhood ${ }^{14}$ : "What do I need to know about you as a person to give you the best care possible?" Supporting patients who are experiencing spiritual and psychological distress is about empathetic, non-judgemental listening, acknowledging and respecting rather than giving advice or religious solace.

Spirituality is the aspect of humanity that refers to the way individuals seek and express meaning and purpose and the way they experience their connectedness to the moment, to self, to others, to nature, and to the significant or sacred.

Following a detailed holistic assessment focusing on the patient's and family's concerns, goals of care can be discussed. The model of shared decision-making is useful in this context. Symptom management aimed at achieving the patients priorities can be started and disease management optimised.

Success in providing primary palliative care depends not only on primary care physicians but also on access to medication and coordination of care. In the case of patients with life-limiting illnesses, we know there is significant suffering, however we do not know the specific experiences of Malaysian patients in their last year of life. We need more research to inform service development. Patients start their illness journey at home in the community, and in most parts of the world, patients want to be cared for close to home. Can we provide the care they need in the place they want to be?

What about care at the end of life when patients are dying? In Malaysia, we still lack data about patients' preferences for care and what they would consider a good death. Studies of patients and carers in both Asian and Western cultures have demonstrated some similarities in what is regarded as important at the end of life, and broad agreement exists regarding pain and symptom control, preparation for death, completion, contributing to others, not being a burden to others and maintaining hope. ${ }^{15}$ Nonetheless, because culture influences the meaning that people give to illness, suffering and dying, we need research that reflects the realities in our communities.

Using the place of death as a marker for quality of care is a topic of debate. Although most patients would like to be at home at the end of life, they prioritise to good symptom control at the end of life. ${ }^{16}$ Palliative care home services with skills in pain and symptom management and access to medication as well as provision of emergency cover can support caring for patients at home. ${ }^{17}$ However, at present, these services 
are only available in urban settings, leaving most patients with no option but to seek care in hospital or suffer in pain.

There are many challenges ahead if patients are to have their palliative care needs addressed in the community, but family medicine physicians and general practitioners are ideally placed to take the first step by identifying patients early, and providing proactive, anticipatory care. Those with positions of influence in policy making can advocate to improve access to services and medication and relieve some of the burden associated with having to access hospital care.

\section{References}

1. World Health Assembly. Resolution WHA67.19 Strengthening of palliative care as a component of comprehensive care throughout the life course. 2014. Accessed October 21,2021. http://apps.who.int/gb/ ebwha/pdf_files/WHA67/A67_R19-en.pdf

2. Zambrano SC, Fliedner MC, Eychmüller $S$. The impact of early palliative care on the quality of care during the last days of life: what does the evidence say?. Curr Opin Support Palliat Care. 2016;10(4):310-315. doi:10.1097/SPC.0000000000000240

3. Qureshi D, Tanuseputro P, Perez R, Pond GR, Seow HY. Early initiation of palliative care is associated with reduced late-life acute-hospital use: A population-based retrospective cohort study. Palliat Med. 2019;33(2):150-159. doi:10.1177/0269216318815794

4. Beernaert K, Deliens L, De Vleminck A, et al. Is There a Need for Early Palliative Care in Patients With LifeLimiting Illnesses? Interview Study With Patients About Experienced Care Needs From Diagnosis Onward. Am J Hosp Palliat Care. 2016;33(5):489-497. doi:10.1177/1049909115577352

5. Hawley PH. The bow tie model of 21 st century palliative care. J Pain Symptom Manage. 2014;47(1):e2-e5. doi:10.1016/j. jpainsymman.2013.10.009
6. Murray SA, Kendall M, Grant E, Boyd K, Barclay S, Sheikh A. Patterns of social, psychological, and spiritual decline toward the end of life in lung cancer and heart failure. J Pain Symptom Manage. 2007;34(4):393-402. doi:10.1016/j.jpainsymman.2006.12.009

7. Munday D, Boyd K, Jeba J, et al. Defining primary palliative care for universal health coverage. Lancet. 2019;394(10199):621-622. doi:10.1016/S0140-6736(19)31830-6

8. The University of Edinburgh. Supportive and Palliative Care Indicators Tool. Accessed November 21, 2021. https://www.spict.org.uk/.

9. Murtagh FE, Ramsenthaler C, Firth A, et al. A brief, patient- and proxy-reported outcome measure in advanced illness: Validity, reliability and responsiveness of the Integrated Palliative care Outcome Scale (IPOS). Palliat Med. 2019;33(8):1045-1057. doi:10.1177/0269216319854264

10. Hui D, Bruera E. The Edmonton Symptom Assessment System 25 Years Later: Past, Present, and Future Developments. J Pain Symptom Manage. 2017;53(3):630-643. doi:10.1016/j.jpainsymman.2016.10.370

11. Ownby KK. Use of the Distress Thermometer in Clinical Practice. J Adv Pract Oncol. 2019;10(2):175-179.
12. Puchalski C, Ferrell B, Virani R, et al. Improving the quality of spiritual care as a dimension of palliative care: the report of the Consensus Conference. J Palliat Med. 2009;12(10):885-904. doi:10.1089/ jpm.2009.0142

13. Chochinov HM. Dignity and the essence of medicine: the A, B, C, and D of dignity conserving care. BMJ. 2007;335(7612):184187. doi:10.1136/bmj.39244.650926.47

14. Chochinov HM, McClement S, Hack T, Thompson G, Dufault B, Harlos M. Eliciting Personhood Within Clinical Practice: Effects on Patients, Families, and Health Care Providers. J Pain Symptom Manage. 2015;49(6):974-80.e2. doi:10.1016/j. jpainsymman.2014.11.291

15. Krikorian A, Maldonado C, Pastrana T. Patient's Perspectives on the Notion of a Good Death: A Systematic Review of the Literature. J Pain Symptom Manage. 2020;59(1):152-164. doi:10.1016/j.jpainsymman.2019.07.033

16. Pollock K. Is home always the best and preferred place of death?. BMJ. 2015;351:h4855. Published 2015 Oct 7. doi:10.1136/bmj.h4855

17. Gomes B, Calanzani N, Curiale V, McCrone P, Higginson IJ. Effectiveness and costeffectiveness of home palliative care services for adults with advanced illness and their caregivers. Cochrane Database Syst Rev. 2013;(6):CD007760. Published 2013 Jun 6. doi:10.1002/14651858.CD007760.pub2 